NASA Technical Memorandum 107415

AIAA-97-0349

\title{
An Acceleration Measurement Capability on International Space Station Supporting Microgravity Science Payloads
}

Thomas J. Sutliff

Lewis Research Center

Cleveland, Ohio

Prepared for the

35th Aerospace Sciences Meeting \& Exhibit

sponsored by the American Institute of Aeronautics and Astronautics

Reno, Nevada, January 6-10, 1997

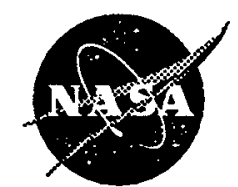

National Aeronautics and

Space Administration 


\title{
AN ACCELERATION MEASUREMENT CAPABILITY ON INTERNATIONAL SPACE STATION SUPPORTING MICROGRAVITY SCIENCE PAYLOADS
}

\author{
Thomas J. Sutliff \\ NASA Lewis Research Center \\ Cleveland, $\mathbf{O H}$
}

\begin{abstract}
The conditions present in low-earth-orbit have enabled various scientific investigations to be conducted. A fundamental reason that space is a useful environment for science is the presence of a decreased effect of gravity while in orbital free-fall. Science investigations are conducted to study phenomena occurring as a result of reductions in buoyancy forces and phenomena enabled as a result of the apparent lack of gravity. The science community requires knowledge of the environment in which their experiments are being conducted. A general purpose measurement system was developed in the 1980's to supply scientific investigators with data of the residual acceleration environment. The Space Acceleration Measurement System, or SAMS, has flown aboard the NASA shuttle fleet over fourteen times, successfully acquiring acceleration measurement data in support of microgravity science experiments.

As NASA continues its development of the International Space Station and its associated research payloads, it once again becomes necessary to address the requirement to measure the residual acceleration in support of the planned scientific investigations. As a follow-on to the SAMS project, SAMS-II was initiated to investigate the requirements and develop the measurement system to support the microgravity science payloads aboard the International Space Station.

The characteristics of the payload complement to be supported by SAMS-II and the different capabilities provided by the station requires a fresh look at the design implementation of a general purpose measurement system. Not only is there a requirement for measurements in support of numerous, potentially simultaneous experiments, but this support is required to be provided for at least ten years of on-orbit operation as compared to a ten to fourteen day mission on shuttle. These differences in fundamental requirements result in the design configuration of the space station-based SAMS-II being significantly
\end{abstract}

different than that of the shuttle-based SAMS.

This paper describes these differences in requirements and the planned features to be provided to the science user community. Some of the unique challenges faced in developing a general purpose measurement system are also discussed.

\begin{tabular}{|c|c|}
\hline \multicolumn{2}{|r|}{ Acronyms } \\
\hline $\mathrm{C} \& \mathrm{DH}$ & ISS Command and Data Handling (network) \\
\hline $\mathrm{CU}$ & SAMS-II Control Unit \\
\hline ESRD & $\begin{array}{l}\text { Experiment Support Requirements } \\
\text { Document }\end{array}$ \\
\hline GOE & SAMS-II Ground Operations Equipment \\
\hline ISS & International Space Station \\
\hline MRL & ISS Medium Rate Link (Ethernet network) \\
\hline OARE & Orbital Acceleration Research Experiment \\
\hline PIMS & $\begin{array}{l}\text { Principal Investigator Microgravity } \\
\text { Services }\end{array}$ \\
\hline RTS & Remote Triaxial Sensor \\
\hline RTS-EE & RTS Electronics Enclosure \\
\hline RTS-SE & RTS Sensor Enclosure \\
\hline SAMS & Space Acceleration Measurement System \\
\hline SAMS-II & A second generation SAMS for ISS \\
\hline TSC & Telescience Support Center \\
\hline
\end{tabular}

Introduction

On-orbit Microgravity Science

Even in the days of the early manned space flight program, scientific experimentation was conducted to take advantage of the unique qualities of orbital conditions. ${ }^{1}$ A fundamental condition occurring as a spacecraft orbits the earth is that it maintains an apparent condition of weightlessness. The spacecraft falls towards the earth at generally the same rate that it is being forced outward from the earth due to its forward motion. An equilibrium condition exists, which has become known as being in microgravity. A simplified viewpoint to understand this concept is to visualize a cannon firing a ball atop a tall mountain. (figure 1) 


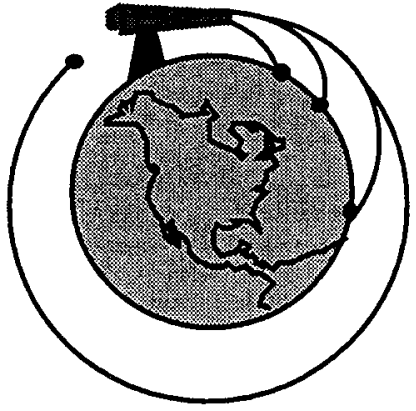

figure 1

Isaac Newton's Cannonball "Thought Experiment"

Once fired, the cannonball falls to earth. As the cannonball is shot at successively faster velocities, it travels farther before landing. Eventually, when shot at the proper velocity, the ball achieves a state of continuous free-fall around the earth. This results in a steady-state condition of free-fall known as an orbit. So, the term microgravity does not represent a fixed magnitude, but rather this condition of equilibrium. ${ }^{2}$

\section{Measurements of the Environment}

As initial scientific experiments were being conducted in microgravity and their results analyzed, the accuracy and reproducibility of research results were called into question. One of the key parameters affecting the outcome, in fact the reason for conducting the science in orbit, was not being measured. That parameter was the accelerations present during the experimentation. Residual accelerations were present and were known to be time varying due to changing conditions such as orbital altitude and vehicle attitude affecting vehicle drag, spacecraft equipment operations, and crewmember activity. In some cases, an experiment apparatus would generate excitations from within which were detrimental to its purpose. Ironically, the assumption of having a quiescent acceleration condition might be violated as a condition of operating the experiment.

At first, numerous independent measurement devices were developed to support individual experiments. As the microgravity science research program matured, a realization was reached that a general purpose measurement system could better satisfy the needs of the science community and reduce duplication of efforts and resources.

\section{Previous Systems}

To satisfy the desire for a general purpose measurement system, a series of instruments known as SAMS, the Space Acceleration Measurement System, were developed at the NASA Lewis Research Center in the late 1980s. ${ }^{3}$ These instruments have now flown over fourteen times, successfully supporting microgravity science experiments conducted aboard the NASA Space Transportation System (shuttle) from 1991 through 1996. One SAMS instrument has been modified and is aboard the Russian space station Mir, acquiring acceleration data in support of cooperative microgravity research activities on that vehicle. ${ }^{4}$ The SAMS instruments acquire acceleration data over the frequency range of $0-100 \mathrm{~Hz}$, although the system has difficulty discerning acceleration magnitudes in the quasi-steady frequency regime, which are of the period of one to fractions of one orbit $(0.0002-0.01 \mathrm{~Hz})$. Another instrument, originally developed to conduct shuttle aerodynamic drag research in low earth orbit, is being successfully used to complement SAMS in this low-frequency regime. The Orbital Aerodynamic Research Experiment (OARE) instrument has flown eight times, specifically supporting five microgravity missions. 5,6 Other systems also have been developed fulfilling unique acceleration measurement needs that a general purpose SAMS instrument could not fulfill.

\section{Requirements Development Process}

The call for a general purpose acceleration measurement system has again been made as NASA and the science community enter a new era in spacebased research using the International Space Station (ISS). In a document discussing the future of microgravity research, the National Research Council has stated the following challenge:

"The $g$-level must be measured accurately, locally, frequently, and synchronously with every experiment."7

A number of fundamental operational differences are anticipated to occur while conducting experiments in a long-duration orbiting facility such as ISS as opposed to those in a relatively short flight-duration platform such as the shuttle. Technology improvements made since SAMS was developed also are resulting in a desire to provide a second generation system for the ISS. The SAMS-II project sets out to improve upon those areas that SAMS and OARE were found through experience to be lacking while, at the same time, leverage the positive feedback from SAMS, OARE, and other successful shuttle-based instruments. A more capable system may be provided to the users as a result of continued advances in technology as well. SAMS-II is required to adapt to and work within a significantly different vehicle than the shuttle, and do so for a considerably longer operational timeframe. Experiences gained from the operation of the SAMS unit aboard Mir continues to provide some insight to 
potential SAMS-II operational approaches on the International Space Station.

The basic requirements set forth for the development of the SAMS-II instrument are formulated from a compilation of user requirements in the science discipline being supported. Microgravity science disciplines of biotechnology, combustion, fluids, low temperature physics, and materials science have specified their peculiar acceleration measurement and data analysis requirements. These discipline-specific requirements are combined into a parent set of system requirements known as the Experiment Support Requirements Document (ESRD). The ESRD states its global requirements for SAMS-II as:

- acquire acceleration data

- measure accelerations with an accuracy and resolution better than the acceleration environment envelope of the ISS program

- acquire the acceleration data with correlated time information

- measure acceleration with selectable frequency range

- measure acceleration in, on and/or near the experiment sample/chamber/apparatus

- allocate control to the users

- principal investigator control of parameters

- on-orbit crew control of parameters

- provide data to users

- supply information in a selectable format

- supply information within a selectable amount of time

The ESRD provides specific guidance to SAMS-II regarding characteristic measurement parameters such as experiment duration, magnitude, and frequency range of interest.

Of course, other factors influence the design and development plans for the SAMS-II including vehicle interface requirements, differing vehicle capabilities than offered by the shuttle, unknowns on the likely environment measurement conditions, likelihood of higher amplitude wideband vibratory energy sources being present, more payloads to be supported, a more "laboratory-type" setting, and potentially more crew time available for research. Of special significance is the likely increase in maturity of the ISS science investigator. The maturing science community is progressing from having the requirement of just being in low gravity to being in a specific environment, to still requiring measurement and verification that the proper test conditions were obtained, to an eventual case in which a user will require environments which are not yet attainable.

Also, the Principal Investigator Microgravity Services (PIMS) project fulfills the responsibility assisting potential microgravity scientists in premission planning by characterizing the general acceleration environment. The PIMS organization responds to requests to describe the environment of a vehicle to support initial scientific feasibility studies and concepts, and aids in the planning for future research on a particular on-orbit platform. The PIMS project is also responsible for maintaining the ESRD based on user requirement inputs.

\section{The SAMS-II Design Evolution}

The process taken to arrive at a successful design solution meeting the requirements for SAMS- $\Pi$ is typical of many aerospace requirements flowdown methods. Basic requirements are used to create a series of conceptual approaches. These approaches are balanced against the requirements in various tradeoff studies, while potential technical questions are answered in key technology areas. A downselection process is conducted at which time the initiation of detailed engineering is begun, eventually leading to development of hardware to be launched to ISS for utilization on-orbit.

Three possible on-orbit system configurations were considered during the conceptual development of SAMS-II.

- fully distributed

- centralized

- hybrid distributed

Each configuration has its own merits and drawbacks.

\section{Fully Distributed}

The concept of a fully distributed system provides maximum coverage potential for desired measurement locations. However, it puts the burden of implementation upon each science payload developer. Some potential advantages include the minimization of power consumption and a decrease in the likely onorbit hardware dedicated to measurement purposes. Unfortunately, with these advantages come significant losses in the service capabilities for consistent data analysis to be provided for all payloads. The PIMS project has a significantly more difficult time gathering consistent data in support of its task of environmental characterization with this approach. Each payload is required to provide its own custom hardware and software interfaces to control and acquire data. The commonality of data analysis tasks also is lost.

Taking this approach relies on each facility to provide commanding, control, data storage, and data routing for this specialized service. Providing such services may result in substantially higher data rate requirements for an individual payload compared to its other experiment parameters. 


\section{Centralized}

A centralized approach was also considered. This approach is much like the SAMS instrument currently provides. Limitations in the ability to reconfigure the system for different measurement needs and the desire by the ISS to not route cables between multiple racks are the primary reasons that this option is not viable.

\section{Hybrid Distributed}

The hybrid combination provides for a centralized control unit which has some "smart" processing capability that can be provided to a payload or investigator on-demand, and commonly developed distributed measurement instruments which are provided to and integrated by each payload developer.

\section{Design Solution}

As a result of inputs from experienced researchers who rely on shuttle-based SAMS and OARE data, and based on tradeoffs of design options for each configuration, the hybrid distributed design scenario was chosen as the basis for the SAMS-II instrument. Key design parameters satisfied from the hybrid configuration center around the versatility to support the needs of users and include:

- multiple payloads are supported over a variable timeline (some on, some off)

- a reconfigurable system responds to changing research desires (maturing user requirements over the ten year operational lifetime of ISS)

- a level of redundancy exists within the system to minimize data disruption or total loss of data

- a data acquisition capability with controllable setup and analysis parameters is responsive to both changing and unknown researcher needs.

During the ISS era of research, facility class payloads which SAMS-II will support plan to service as many as ten investigators per year. Generally, each investigator will have his/her own preferences on the measurement system configuration. Such user-based desires lead SAMS-II toward an adaptable configuration such as is available with the hybrid design solution.

Functions being provided by SAMS-II include the ability for a principal investigator to control the acquisition of acceleration data for his experiment and to analyze the data in near-real-time on-board the ISS. Options being implemented include adjustable frequency ranges and selectable data analysis parameters ranging from peak and rms value calculations to acceleration versus frequency versus time spectrograms.

\section{Data Acquisition}

A variable acquisition rate (or usable frequency range) will provide researchers control of acquisition conditions to allow adaptation to changing measurement needs.

A wide dynamic range analog-to-digital converter is provided. Doing so eliminates the need for manual or automatic gain switches, and eliminates the data loss associated with the switching periods.

The analog acceleration measurements is digitized within the measurement head. Digitizing the data as close to the measurement transducer as possible provides the highest likelihood of good data quality by minimizing noise source encroachment on the signal.

\section{Data Analysis}

On-board data analysis allows investigators to make smart operational decisions and provides a mechanism for information feedback to payloads conducting their science. For example, an investigator may require knowledge of the recent microgravity history prior to initiation of a critical portion of an experiment or may only need to monitor that the magnitude of the acceleration environment remains below an acceptable threshold level during the experiment operation. Other users might require receipt of a deviation report or possibly initiate data storage if the threshold is exceeded.

For the user who requires a more detailed view of the data, processing routines are provided. Standard data analysis tools are currently in use for the analysis of SAMS and OARE data. ${ }^{8}$ These basic tools are incorporated within the on-orbit SAMS-II instrument as optional functions, allowing researchers the ability to conduct high-level data processing on-orbit. The advantage of optional on-orbit data analysis is that informational feedback to their payload is feasible and the need for downlink bandwidth decreases as a result of creating a reduced size information data set.

\section{Crew Interaction}

A crew display is provided allowing the crew to interact with the SAMS-II as a user and to understand the ramifications of their nominal activities in producing disturbances to the microgravity environment. The crew also will benefit from the onboard data processing capability defined above.

\section{Maintainability}

An additional design driver for the SAMS-II instrument is that it be maintainable. As a general purpose system planned for ten years of on-orbit use, the instrument is expected to be capable of periodic technology enhancements, and will likely require occasional servicing. A design solution addressing the capability for modular, incremental improvements is an ongoing project goal. 


\section{Operational Considerations}

All these performance capabilities must be balanced against a desire to provide a relatively straightforward operational system. The SAMS-II design efforts continue to focus on the user. The plan is to provide users with direct control over a dedicated portion of the SAMS-II operational capability. Investigators using the SAMS-II capabilities are primarily interested in the results of their science, such as their fluids or combustion experiment data. They are not expected to be experts in the field of acceleration measurement and data processing. To accommodate the average investigator, default acquisition and data analysis parameters are provided to allow meaningful data to be acquired easily, while also permitting a variable command structure user interface to be formulated. Such an interface provides an investigator with options in acquiring and analyzing the acceleration information in the most meaningful way in reference to the science experiment being conducted.

Specific features provided to the science user community to enhance their operational capabilities include:

- commanding of acquisition parameters

- data analysis (interactive selections)

- on-board display (crew as researcher)

- selection of downlink and/or on-board data storage for subsequent retrieval of data following resupply mission.

\section{Other Design Considerations}

Some additional challenges the SAMS-II project faces in development of a general purpose measurement system for the ISS include:

- development of a system prior to the assignment of specific investigators

- soft system (ISS) interfaces during parallel development of the vehicle and SAMS-II

- an unknown measurement environment.

As a result of these challenges, numerous other considerations are being taken into account during the preliminary design of the SAMS-II instrument. Since the SAMS-II and ISS are in development concurrently, SAMS-II continues to adapt to the ISS vehicle capability and occasionally compromises away from the preferred approach in order to reach a viable overall solution. To minimize difficulty in these transitions, a philosophy has been adopted which fosters design flexibility to enable this responsiveness to change.

Use of Commercial-Off-the-Shelf (COTS) technology allows a standard building block approach to be taken. The primary advantage to the use of COTS hardware is that although the best configurations available now are used, they will be obsolete within a year after placement in service and subsequent upgrades are possible later if changing requirements warrant additional capability. This approach results in an upgrade path in future, and provides for known benchmark performance of components. An additional advantage to the use of COTS is that numerous development platforms and tools are available, easing the cost of custom hardware/software development.

\section{SAMS-II Design Configuration}

The on-orbit hardware for the SAMS-II hybrid modular design consists of a Control Unit (CU), multiple Remote Triaxial Sensors (RTS), and network connections between the CU and RTS hardware using ISS resources. A block diagram overview of the key elements of the on-orbit system is shown in figure 2 .

The CU houses the common data acquisition and analysis functions of SAMS-II such as data processing and mass storage systems. The primary subsystems include the electronics card cage which houses a central processor unit as well as communications, graphics display, and health monitoring circuitry. A mass storage subsystem is provided consisting of a hard drive and a removable media optical drive. Support subsystems for the $\mathrm{CU}$ include the power and thermal control subsystems and the structural enclosure subsystem.

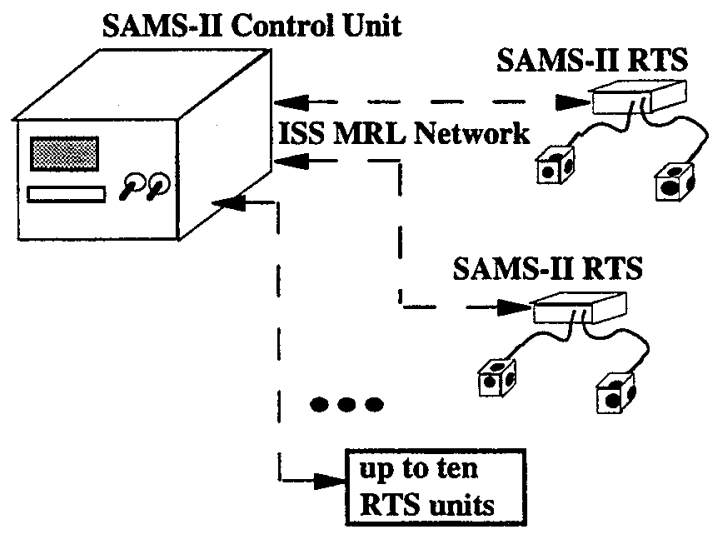

figure 2

On-orbit SAMS-II Block Diagram Configuration

A quasi-steady acceleration sensor subsystem whose data is accessible by all users also resides within the $\mathrm{CU}$. To perform the quasi-steady measurement, an electrostatically suspended mass is floated within a constrainment loop. Occasional bias calibrations are conducted by inverting the transducer axes, one at a time. This operation is similar to that provided by the 
OARE system. ${ }^{5}$ The Control Unit functions as the intermediary for the RTS measurement systems and the on-orbit payloads and their science users.

Multiple RTS systems are distributed throughout the vehicle as near to the location of research as possible within the constraints of RTS size and experiment operational parameters. The locations of the sensor units are chosen to provide a true measurement of the environment present at the location of the science investigation. The RTS system is comprised of two primary elements- the Electronics Enclosure (-EE) and the Sensor Enclosure (-SE). Each RTS-EE provides the network interface to the CU and the power and data commanding interface to two RTS-SE units. Each RTS-SE contains a set of three orthogonal servo accelerometers, signal conditioning circuitry and digitizing electronics.

Communication between each RTS and the CU is provided by the Command and Data Handling (C\&DH) network aboard the ISS. Based on data rate estimates, the Medium Rate Link (MRL) is utilized. This MRL operates via an Ethernet protocol.

Once the multiple RTS data streams are received and optionally analyzed by the $\mathrm{CU}$, a bundled data stream may be retransmitted to the ground via the ISS downlink. This data stream then is routed to the Telescience Support Center (TSC) at the Lewis Research Center where the SAMS-II Ground Operations Equipment (GOE) catalogs the operational information, and provides the desired data to the specific research investigator either locally at the TSC or at a remote site. (figure 3 )

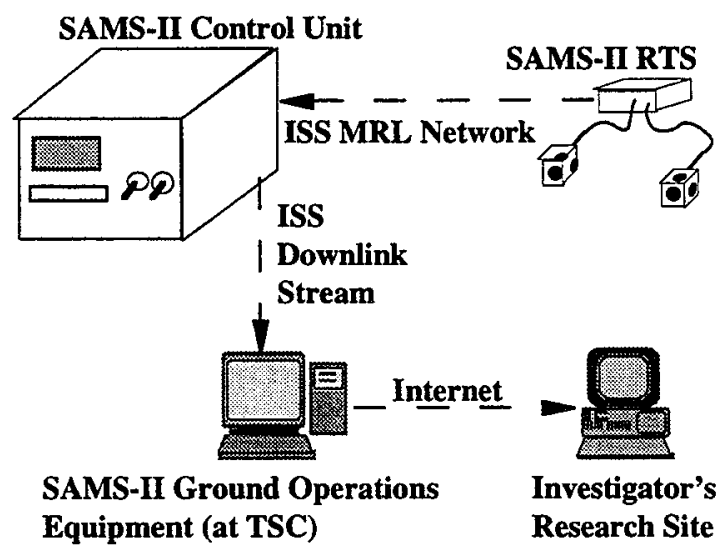

figure 3

Data Transmission Flow Diagram

The investigator may choose to store or interpret this data as part of their science investigation. It is also possible for the user to adjust acquisition parameters and have the new configuration routed to the uplink, to the CU and subsequently to the RTS allocated to the payload, where the adjustment in acquisition parameter takes place. Analysis parameters are similarly adjustable within the CU by the user.

\section{Conclusion}

The SAMS-II instrument is being developed with four key parameters in mind. They all focus on the common theme of maintaining the operational user as their customer.

- use of lessons learned and researcher feedback to drive the design capabilities

- a knowledge that the system will be required to respond to requirements changes throughout the lifetime of the research conducted on ISS

- use of commonly available technology and a modular design to enable future enhancements

- an improvement on the combination of the capabilities of SAMS and OARE in support of multiple researchers and areas of research having widely varying acceleration measurement requirements

Thus, the SAMS-II instrument provides a versatile acceleration measurement system for use aboard the International Space Station by the microgravity science community.

\section{References}

1. Petrash, D. A., Nussle, et. al.; Effect of Acceleration Disturbances Encountered in MA-7 Spacecraft on the Liquid-Vapor Interface, NASA TN D-1577, 1963.

2. A Microgravity Investigator's Guide, NASA Microgravity Science and Applications Division, 1994.

3. DeLombard, R.; Finley; et. al.; Development of and Flight Results from the Space Acceleration Measurement System (SAMS); NASA TM-105652 (AIAA 92-0354), 1992.

4. DeLombard, R. and Rogers, M. J. B.; Quick Look Report of Acceleration Measurements on Mir Space Station During Mir-16, NASA TM-106835, 1995.

5. Blanchard, R. C., Nicholson, et. al.; Qrbital Acceleration Research Experiment: Calibration Measurements, Journal of Spacecraft and Rockets, Vol. 32, No. 4, pp. 619-626, 1995.

6. Wagar, W. O., Lugasy, et. al.; A Plan for Orbital Acceleration Research Experiment Operations on STS-73 (USML-2), AIAA 95-0689, 1995.

7. Toward a Microgravity Research Strategy, Space Studies Board, National Research Council, 1992.

8. DeLombard, R.; Compendium of Information for Interpreting the Microgravity Environment of the Orbiter Spacecraft; NASA TM-107032, 1996. 
Public reporting burden for this collection of information is estimated to average 1 hour per response, including the time for reviewing instructions, searching existing data sources, gathering and maimtaining the data needed, and completing and reviewing the collection of information. Send comments regarding this burden estimate or any other aspect of this collection of Information, including suggestions for reducing this burden, to Washington Headquarters Services, Dhectorate for information Coperations and Reports, 2015 .

\begin{tabular}{|l|l|l} 
1. AGENCY USE ONLY (Leave blank) & $\begin{array}{c}\text { 2. REPORT DATE } \\
\text { February } 1997\end{array}$ & $\begin{array}{r}\text { 3. REPOAT TYPE AND DATES COVERED } \\
\text { Technical Memorandum }\end{array}$
\end{tabular}

\section{TITLE AND SUBTIILE}

An Acceleration Measurement Capability on International Space Station Supporting Microgravity Science Payloads

6. AUTHOR(S)

Thomas J. Sutliff
5. FUNDING NUMBERS

WU-963-60-0B
7. PERFOAMING ORGANIZATION NAME(S) AND ADDRESS(ES)

National Aeronautics and Space Administration

Lewis Research Center

Cleveland, Ohio 44135-3191
8. PERForming organization REPORT NUMBER

E-10652
9. SPONSORING/MONITORING AGENCY NAME(S) AND ADDRESS(ES)

National Aeronautics and Space Administration

Washington, DC 20546-0001
10. SPONSORING/MONITORING AGENCY REPORT NUMBER

NASA TM-107415

AIAA-97-0349

11. SUPPLEMENTARY NOTES

Prepared for the 35th Aerospace Sciences Meeting \& Exhibit sponsored by the American Institute of Aeronautics and Astronautics, Reno, Nevada, January 6-10, 1997. Responsible person, Thomas J. Sutliff, organization code 6743, (216) 433-3887.

12a. DISTRIBUTIONAVALABILTY STATEMENT

12b. DISTRIBUTION CODE

Unclassified -Unlimited

Subject Categories 19, 29, 35, and 62

This publication is available from the NASA Center for AeroSpace Information, (301) 621-0390.

13. ABSTRACT (Maximum 200 words)

The conditions present in low-earth-orbit have enabled various scientific investigations to be conducted. A fundamental reason that space is a useful environment for science is the presence of a decreased effect of gravity while in orbital free-fall. Science investigations are conducted to study phenomena occurring as a result of reductions in buoyancy forces and phenomena enabled as a result of the apparent lack of gravity. The science community requires knowledge of the environment in which their experiments are being conducted. A general purpose measurement system was developed in the 1980's to supply scientific investigators with data of the residual acceleration environment. The Space Acceleration Measurement System, or SAMS, has flown aboard the NASA shuttle fleet over fourteen times, successfully acquiring acceleration measurement data in support of microgravity science experiments. As NASA continues its development of the Intemational Space Station and its associated research payloads, it once again becomes necessary to address the requirement to measure the residual acceleration in support of the planned scientific investigations. As a follow-on to the SAMS project, SAMS-II was initiated to investigate the requirements and develop the measurement system to support the microgravity science payloads aboard the International Space Station. The characteristics of the payload complement to be supported by SAMS-II and the different capabilities provided by the station requires a fresh look at the design implementation of a general purpose measurement system. Not only is there a requirement for measurements in support of numerous, potentially simultaneous experiments, but this support is required to be provided for at least ten years of on-orbit operation as compared to a ten to fourteen day mission on shuttle. These differences in fundamental requirements result in the design configuration of the space station-based SAMS-II being significantly different than that of the shuttle-based SAMS. This paper describes these differences in requirements and the planned features to be provided to the science user community. Some of the unique challenges faced in developing a general purpose measurement system are also discussed.

14. SUBJECT TERMS

Microgravity; Acceleration; International Space Station; Measurement; Telescience 15. NUMBER OF PAGES 8

\begin{tabular}{|c|c|}
\hline $\begin{array}{c}\text { 17. SECURTY CLASSIFICATION } \\
\text { OF REPORT } \\
\text { Unclassified }\end{array}$ & $\begin{array}{c}\text { 18. SECURTYY CLASSIFICATION } \\
\text { OF THIS PAGE } \\
\text { Unclassified }\end{array}$ \\
\hline
\end{tabular}

NSN 7540-01-280-5500
19. SECURITY CLASSIFICATION OF ABSTRACT Unclassified 

National Aeronautics and

Space Administration

Lewis Research Center

21000 Brookpark Rd.

Cleveland, $\mathrm{OH}$ 44135-3191

Offlclal Business

Penally for Private Use $\$ 300$

POSTMASTER: If Undeliverable - Do Not Return 